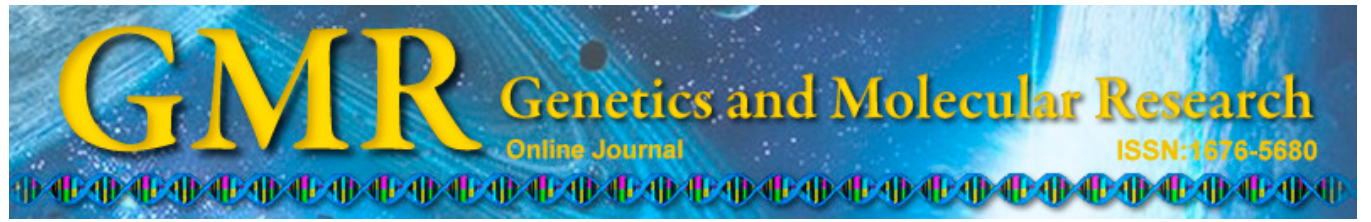

\title{
Arg188His polymorphism in the $X R C C 2$ gene and the risk of ovarian cancer: a meta-analysis
}

\author{
M. Zhai ${ }^{1}$,Y. Wang ${ }^{1}$ and M.F. Jiang ${ }^{2}$ \\ ${ }^{1}$ Comprehensive Laboratory, \\ Ningbo International Travel Healthcare Center, Ningbo, China \\ ${ }^{2}$ Laboratory Department, Hangzhou Cancer Hospital, \\ Hangzhou, China \\ Corresponding author: M.F. Jiang \\ E-mail: jiangmf001@126.com
}

Genet. Mol. Res. 14 (3): 10808-10815 (2015)

Received February 12, 2015

Accepted May 28, 2015

Published September 9, 2015

DOI http://dx.doi.org/10.4238/2015.September.9.19

\begin{abstract}
Numerous studies have evaluated the association between the Arg188His polymorphism of the X-ray repair cross-complementing group 2 (XRCC2) gene and ovarian cancer risk. However, the specific association is still controversial. This meta-analysis was therefore designed to clarify these controversies. Relevant case-control studies were enrolled in the meta-analysis. Quality evaluation of the included studies was conducted by two physicians. Statistical analyses were carried out using the Stata 12.0 software for meta-analysis. Analyses of sensitivity and publication bias were also conducted. Overall, a significant association was found between the Arg 188 His polymorphism and ovarian cancer risk when all studies were pooled into the metaanalysis (Arg/Arg vs His/His: $\mathrm{OR}=1.85,95 \% \mathrm{CI}=1.15-3.00 ; \mathrm{Arg} / \mathrm{Arg}$ vs $\mathrm{Arg} / \mathrm{His}: \mathrm{OR}=1.17,95 \% \mathrm{CI}=1.03-1.32$; dominant model: $\mathrm{OR}=0.84$, $95 \% \mathrm{CI}=0.74-0.95$; recessive model: $\mathrm{OR}=1.69,95 \% \mathrm{CI}=1.05-2.70$ ). This meta-analysis suggested that the XRCC2 Arg188His polymorphism was associated with the risk of ovarian cancer. Further large and welldesigned studies are needed to confirm these conclusions.
\end{abstract}

Key words: X-ray repair cross-complementing group 2 (XRCC2); Gene polymorphism; Arg188His; Ovarian cancer 


\section{INTRODUCTION}

Ovarian cancer is one of the most common gynecological malignancies among women, especially those aged $\geq 50$ years (Ferlay et al., 2010). Overall, $75 \%$ of patients are diagnosed at an advanced stage and consequently the 5 -year survival rate is poor, since ovarian cancer is generally asymptomatic in its early stages and there is currently no effective screening method (Chang et al., 2011). Currently, the etiology and pathogenesis of ovarian cancer remain unclear. Parity, oral contraceptive use, and family history are well-known risk factors for ovarian cancer. In addition, the pathogenesis of ovarian cancer is thought to be influenced by multiple genetic factors, and many reports have highlighted the investigation of genes underlying the development and progression of ovarian cancer (Bae et al., 2014; Jiang et al., 2014).

DNA repair systems play critical roles in protecting against mutations and are essential for maintaining the integrity of the genome. To date, more than a hundred proteins implicated in DNA repair have been found in human cells. These proteins are implicated in five major DNA repair pathways, including nucleotide excision repair, base excision repair, mismatch repair, homologous recombination repair, and non-homologous end-joining (Wood et al., 2001). The X-ray repair cross-complementing 2 (XRCC2) protein encodes a member of the $\mathrm{RecA} / \operatorname{Rad} 51-$ related protein family that participates in the homologous recombination repair pathway to maintain chromosome stability and repair DNA damage (Braybrooke et al., 2000).

The $X R C C 2$ gene, located at 7q36.1, contains a $\mathrm{G}$ to A polymorphism located in exon 3 resulting in a substitution of histidine (His) for arginine (Arg) (Arg188His) (Yu et al., 2010). In the past decade, a number of epidemiological studies have assessed the association between the Arg188His polymorphism in the XRCC2 gene and ovarian cancer risk. However, the results have been inconsistent. Meta-analysis can be a useful tool in detecting an association that could otherwise remain masked in studies with smaller sample size, especially in those evaluating rare allele frequency polymorphisms. The aim of this meta-analysis was to investigate the association between the XRCC2 $\operatorname{Arg} 188$ His polymorphism and the susceptibility to ovarian cancer in all eligible case-control studies published to date.

\section{MATERIAL AND METHODS}

\section{Literature search strategy}

Study retrieval was conducted in PubMed and EMBASE databases using the terms "X-ray repair cross-complementing 2", "XRCC2", "meta analysis", "Arg188His", and "ovarian cancer" through June, 2014. The reference lists of major textbooks, reviews, and included articles were examined through manual searches to find other potentially eligible studies. Studies reported by the same authors, although published in different journals, were checked for possible overlapping participant groups. When pertinent data were not included, or data that were presented were unclear, the authors were contacted directly.

\section{Inclusion and exclusion criteria}

The inclusion criteria comprised: 1) case-control studies that addressed patients with ovarian cancer and healthy controls; 2) studies on the association of the XRCC2 Arg188His 
polymorphism and susceptibility to ovarian cancer; 3) essential information about the distribution on genotype frequency was described in detail, and the data were extractable; 4) there were detailed descriptions of study subject and DNA sources; and 5) the authors provided sample size, ORs and $95 \% \mathrm{CIs}$. The exclusion criteria comprised: 1) not case-control studies that evaluated the association between the XRCC2 Arg188His polymorphism and ovarian cancer risk; 2) case reports, letters, reviews, meta-analyses, and editorial articles; 3) reports in which the number of null- and wild-type genotypes could not be ascertained; 4) inclusion of duplicate data in the studies; and 5) lack of essential information. Additionally, we selected the most recent study when some reports were repeated.

\section{Data extraction}

Two investigators (M. Zhai and Y. Wang) independently extracted and checked the information; consensus was reached by discussion. From each of the included articles, the following information was extracted: first author, year of publication, area, numbers of patients and controls, distributions of genotypes and alleles, and evidence of Hardy-Weinberg equilibrium (HWE) (Table 1).

\section{Statistical analysis}

For each study, HWE was analyzed with a chi-square-based test. We calculated the ORs and corresponding 95\%CIs to evaluate the association between the XRCC2 Arg188His polymorphism and ovarian cancer risk under a homozygote comparison (Arg/Arg vs His/His), a heterozygote comparison (Arg/Arg vs Arg/His), a dominant model (His/His + Arg/His vs Arg/Arg), and a recessive model (Arg/Arg + Arg/His vs His/His) between groups. The effect of heterogeneity was quantified using $\mathrm{I}^{2}$, which ranges between 0 and $100 \%$ and represents the proportion of study variability attributable to heterogeneity rather than chance. $I^{2}$ values of 25,50 , and $75 \%$ were nominally defined as low, moderate, and high estimates, respectively. When $\mathrm{I}^{2}>50 \%$ indicated heterogeneity across studies, the random-effect model was used for meta-analysis; else the fixed-effect model was calculated. Sensitivity analysis was mainly performed by sequential omission of individual studies or non-HWE studies. Publication bias was investigated by funnel plot and Begg's funnel plot. All statistical analyses were performed by using STATA version 12.0 (Stata Corporation, College Station, TX, USA).

\section{RESULTS}

\section{Characteristics of eligible studies}

The search strategy retrieved 35 potentially relevant articles. Base on the inclusion criteria, 8 case-control studies from 4 publications were included in this meta-analysis (Auranen et al., 2005; Webb et al., 2005; Beesley et al., 2007; Mohamed et al., 2013). The flow chart for study selection is summarized in Figure 1. The 8 case-control studies selected included a total of 3565 patients and 6604 healthy controls, and were published between 20052013. All the articles were written in English and were based on healthy individuals, and all were performed in Caucasians. The HWE test was performed on the genotype distributions of 
the controls; all studies were in HWE except Mohamed et al. (2013). In addition, the controls of all the eligible studies were taken from the general populations. The genotype distributions and risk allele frequencies are summarized in Table 1.



Figure 1. Flow diagram of the study search and selection process.

\begin{tabular}{|c|c|c|c|c|c|c|c|c|c|c|}
\hline \multirow[t]{2}{*}{ Study } & \multirow[t]{2}{*}{ Area } & \multirow[t]{2}{*}{ Race } & \multirow[t]{2}{*}{ Patients/Controls } & \multicolumn{3}{|c|}{ Genotypes for patients } & \multicolumn{3}{|c|}{ Genotypes for controls } & \multirow[t]{2}{*}{ HWE test } \\
\hline & & & & Arg/Arg & $\mathrm{Arg} / \mathrm{His}$ & His/His & Arg/Arg & Arg/His & His/His & \\
\hline Auranen et al. (2005) & UK & Caucasian & $729 / 842$ & 629 & 98 & 2 & 704 & 129 & 9 & 0.26 \\
\hline Auranen et al. (2005) & Denmark & Caucasian & $269 / 561$ & 238 & 31 & 0 & 484 & 75 & 2 & 0.61 \\
\hline Auranen et al. (2005) & US & Caucasian & $315 / 404$ & 260 & 54 & 1 & 331 & 68 & 5 & 0.48 \\
\hline Auranen et al. (2005) & UK & Caucasian & $275 / 1811$ & 251 & 23 & 1 & 1538 & 267 & 6 & 0.11 \\
\hline Webb et al. (2005) & Australia & Caucasian & $524 / 1118$ & 451 & 68 & 5 & 952 & 156 & 10 & 0.20 \\
\hline Webb et al. (2005) & Australia & Caucasian & $430 / 950$ & 364 & 63 & 3 & 802 & 140 & 8 & 0.49 \\
\hline Beesley et al. (2007) & Australia & Caucasian & $923 / 818$ & 799 & 117 & 7 & 696 & 115 & 7 & 0.35 \\
\hline Mohamed et al. (2013) & Egypt & Caucasian & $100 / 100$ & 36 & 58 & 6 & 24 & 60 & 16 & 0.03 \\
\hline
\end{tabular}

HWE $=$ Hardy-Weinberg equilibrium.

\section{Quantitative synthesis}

The evaluation of the association between the Arg188His polymorphism and the risk of ovarian cancer is displayed in Figure 2 and in Table 2. Meta-analysis results showed significant associations between the Arg188His polymorphism and ovarian cancer risk (Arg/ Arg $v s$ His/His: $\mathrm{OR}=1.85,95 \% \mathrm{CI}=1.15-3.00 ; \mathrm{Arg} / \mathrm{Arg} v s \mathrm{Arg} / \mathrm{His}: \mathrm{OR}=1.17,95 \% \mathrm{CI}=$ 1.03-1.32; dominant model: $\mathrm{OR}=0.84,95 \% \mathrm{CI}=0.74-0.95$; recessive model: $\mathrm{OR}=1.69$, 
$95 \% \mathrm{CI}=1.05-2.70)$. Sensitivity analysis was performed by removing the study by Mohamed et al. (2013) due to the genotype distribution in the control group therein deviating from HWE; from this, the pooled OR and P value for the overall effect of the null-genotype did not significantly change, suggesting that the results of meta-analysis were statistically robust (Arg/Arg vs His/His: OR = 1.77 95\%CI = 1.08-2.88; Arg/Arg vs Arg/His: OR = 1.16, 95\%CI $=1.02-1.32$; dominant model: $\mathrm{OR}=0.84,95 \% \mathrm{CI}=0.74-0.96$; recessive model: $\mathrm{OR}=1.59$, $95 \% \mathrm{CI}=0.99-2.58)$.

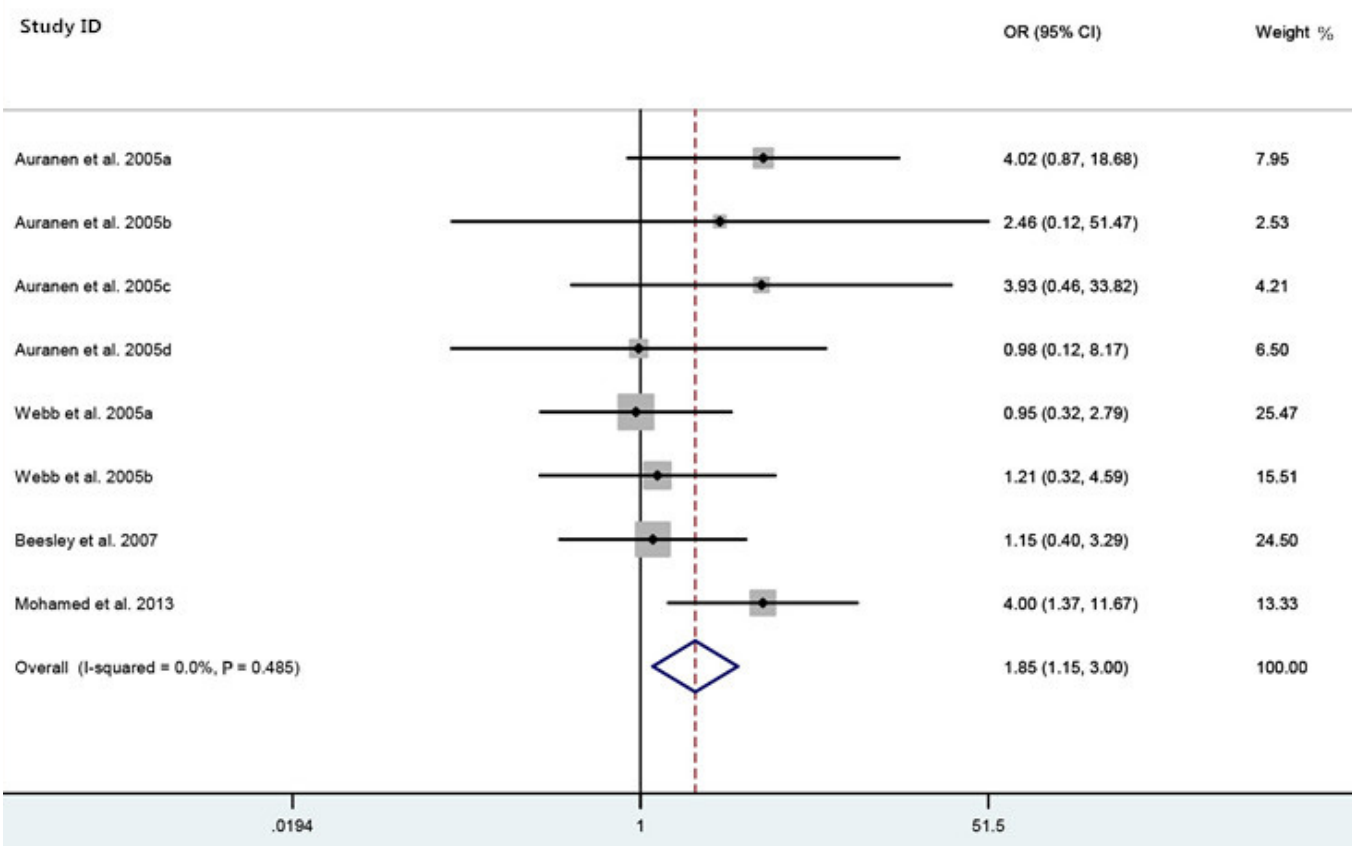

Figure 2. Forest plot of ovarian cancer risk associated with the XRCC2 Arg188His polymorphism in the Arg/Arg vs His/His model.

Table 2. Summary ORs and $95 \%$ CIs of the $X R C C 2 \operatorname{Arg} 188$ His polymorphism and ovarian cancer risk.

\begin{tabular}{|c|c|c|c|c|c|c|c|c|c|c|}
\hline \multirow[t]{2}{*}{ Subgroup } & \multirow[t]{2}{*}{ Genetic model } & \multicolumn{2}{|c|}{ Sample size } & \multirow[t]{2}{*}{$\begin{array}{l}\text { Type of } \\
\text { model }\end{array}$} & \multicolumn{2}{|c|}{$\begin{array}{c}\text { Test of } \\
\text { heterogeneity }\end{array}$} & \multicolumn{2}{|c|}{$\begin{array}{c}\text { Test of } \\
\text { association }\end{array}$} & \multicolumn{2}{|c|}{$\begin{array}{c}\text { Test of } \\
\text { publication bias }\end{array}$} \\
\hline & & Patients & Controls & & $I^{2}$ & $\mathrm{P}$ & OR & $95 \% \mathrm{CI}$ & $z$ & $\mathrm{P}$ \\
\hline \multirow[t]{4}{*}{ Overall } & Arg/Arg vs His/His & 3565 & 6604 & Fixed & $0.0 \%$ & 0.49 & 1.85 & $1.15-3.00$ & 0.00 & 1.00 \\
\hline & $\mathrm{Arg} / \mathrm{Arg}$ vs $\mathrm{Arg} / \mathrm{His}$ & & & Fixed & $1.0 \%$ & 0.42 & 1.17 & $1.03-1.32$ & 0.00 & 1.00 \\
\hline & Dominant model & & & Fixed & $8.7 \%$ & 0.36 & 0.84 & $0.74-0.95$ & 0.00 & 1.00 \\
\hline & Recessive model & & & Fixed & $0.0 \%$ & 0.51 & 1.69 & $1.05-2.70$ & 0.00 & 1.00 \\
\hline Consistent with & Arg/Arg vs His/His & 3465 & 6504 & Fixed & $0.0 \%$ & 0.72 & 1.77 & $1.08-2.88$ & 0.00 & 1.00 \\
\hline \multirow{3}{*}{ HWE } & Arg/Arg vs Arg/His & & & Fixed & $3.6 \%$ & 0.40 & 1.16 & $1.02-1.32$ & 0.00 & 1.00 \\
\hline & Dominant model & & & Fixed & $0.0 \%$ & 0.43 & 0.84 & $0.74-0.96$ & 0.00 & 1.00 \\
\hline & Recessive model & & & Fixed & $0.0 \%$ & 0.51 & 1.59 & $0.99-2.58$ & 0.00 & 1.00 \\
\hline
\end{tabular}

HWE $=$ Hardy-Weinberg equilibrium. 


\section{Publication bias}

The funnel plot and the Begg test were used to assess the publication bias. There was no evidence of publication bias in our study (Figure 3 and Table 2). The results implied that the publication bias was low in the present meta-analysis.



Figure 3. Begg funnel plot test of publication bias for the association of the $X R C C 2 \mathrm{Arg} 188 \mathrm{His}$ polymorphism and ovarian cancer in the Arg/Arg vs His/His model.

\section{DISCUSSION}

Ovarian cancer is the third most common tumor of the female genital tract after carcinomas of the cervix and endometrium and remains the leading cause of gynecological malignancy-related mortality (Siegel et al., 2011). It is difficult to detect ovarian cancer at its early stages using conventional methods. Therefore, there is a need for biomarkers of higher diagnostic accuracy to distinguish malignant from benign pelvic masses at an early stage and to set up an effective screening program. Genetic polymorphisms altering the level of protein expressed would be anticipated to have a substantial influence on disease activity (Tahara et al., 2009). Recently various studies have focused on the association between the XRCC2 gene Arg188His polymorphism and ovarian cancer risk. However, the results have been controversial, in part because of the possibly small effect of the polymorphism on cancer risk and of the relatively small sample size in each of published studies (Danoy et al., 2007; Du et al., 2013; Zhu et al., 2013). To clarify the controversial findings, we conducted this meta-analysis.

Our meta-analysis quantitatively assessed the association between the Arg188His polymorphism in the XRCC2 gene and ovarian cancer risk. To the best of our knowledge, this is the first meta-analysis assessing the relationship between the Arg188His polymorphism 
and the risk of ovarian cancer. Overall, 8 case-control studies from 4 articles were included and assessed, involving a total of 3465 patients and 6504 healthy controls, and the results revealed that the Arg188His polymorphism in the XRCC2 gene was significantly associated with the susceptibility to ovarian cancer. Further sensitivity analysis confirmed the significant association between the Arg188His polymorphism in the XRCC2 gene and ovarian cancer risk. No evidence was found supporting publication bias in this meta-analysis. The mechanism underlying the $X R C C 2$ gene Arg188His polymorphism relationship to ovarian cancer risk is still unclear. A previous study showed that XRCC2 (Arg188His), ERCC2 (K751Q), and $C D K N 1 B$ (V109G) variants could synergistically contribute to increased ovarian cancer risk. Further studies of gene-environment interactions should be taken into consideration to get a better, more comprehensive understanding of the association (Lin et al., 2013).

There are some limitations to this meta-analysis. First, because of incomplete raw data, some relevant studies could not be included in our analysis. Second, our systematic review was based on unadjusted data, as genotype information stratified for the main confounding variables was not available in the original papers and the confounding factors addressed across the different studies were variable. Finally, some valuable studies were probably omitted by the impact of publication bias, because articles with positive results or in English were more likely to have been published. Therefore, future studies should include a larger sample size, rigorous design approach, perfect retrieval strategy, and reasonable inclusion and exclusion criteria.

In conclusion, this meta-analysis determined that the XRCC2 gene Arg188His polymorphism might be associated with ovarian cancer risk. Further studies estimating the effect of gene-gene and gene-environment interactions might eventually provide a better, more comprehensive understanding of this association.

\section{Conflicts of interest}

The authors declare no conflict of interest.

\section{REFERENCES}

Auranen A, Song H, Waterfall C, Dicioccio RA, et al. (2005). Polymorphisms in DNA repair genes and epithelial ovarian cancer risk. Int. J. Cancer 117: 611-618.

Bae HS, Kim HJ, Hong JH, Lee JK, et al. (2014). Obesity and epithelial ovarian cancer survival: a systematic review and meta-analysis. J. Ovarian Res. 7: 41.

Beesley J, Jordan SJ, Spurdle AB, Song H, et al. (2007). Association between single-nucleotide polymorphisms in hormone metabolism and DNA repair genes and epithelial ovarian cancer: results from two Australian studies and an additional validation set. Cancer Epidemiol. Biomarkers Prev. 16: 2557-2565.

Braybrooke JP, Spink KG, Thacker J and Hickson ID (2000). The RAD51 family member, RAD51L3, is a DNA-stimulated ATPase that forms a complex with XRCC2. J. Biol. Chem. 275: 29100-29106.

Chang X, Ye X, Dong L, Cheng H, et al. (2011). Human epididymis protein 4 (HE4) as a serum tumor biomarker in patients with ovarian carcinoma. Int. J. Gynecol. Cancer 21: 852-858.

Danoy P, Sonoda E, Lathrop M, Takeda S, et al. (2007). A naturally occurring genetic variant of human XRCC2 (R188H) confers increased resistance to cisplatin-induced DNA damage. Biochem. Biophys. Res. Commun. 352: 763-768.

Du J, Lu C, Cui G, Chen Y, et al. (2013). DNA repair gene XRCC1 polymorphisms and susceptibility to childhood acute lymphoblastic leukemia: a meta-analysis. Chin. J. Cancer Res. 25: 405-415.

Ferlay J, Shin HR, Bray F, Forman D, et al. (2010). Estimates of worldwide burden of cancer in 2008: GLOBOCAN. Int. J. Cancer 127: 2893-2917.

Jiang PY, Jiang ZB, Shen KX and Yue Y (2014). Fish intake and ovarian cancer risk: a meta-analysis of 15 case-control and cohort studies. PLoS One 9: e94601. 
Lin G, Zhao J, Wu J, Andreevich OR, et al. (2013). Contribution of catechol-O-methyltransferase Val158Met polymorphism to endometrial cancer risk in postmenopausal women: a meta-analysis. Genet. Mol. Res. 12: 6442-6453.

Mohamed FZ, Hussien YM, AlBakry MM, Mohamed RH, et al. (2013). Role of DNA repair and cell cycle control genes in ovarian cancer susceptibility. Mol. Biol. Rep. 40: 3757-3768.

Siegel R, Ward E, Brawley O and Jemal A (2011). Cancer statistics, 2011: the impact of eliminating socioeconomic and racial disparities on premature cancer deaths. CA Cancer J. Clin. 61: 212-236.

Tahara T, Shibata T, Nakamura M, Yamashita H, et al. (2009). Effect of polymorphisms in the 3' untranslated region (3'UTR) of vascular endothelial growth factor gene on gastric cancer and peptic ulcer diseases in Japan. Mol. Carcinog. 48: 1030-1037.

Webb PM, Hopper JL, Newman B, Chen X, et al. (2005). Double-strand break repair gene polymorphisms and risk of breast or ovarian cancer. Cancer Epidemiol. Biomarkers Prev. 14: 319-323.

Wood RD, Mitchell M, Sgouros J and Lindahl T (2001). Human DNA repair genes. Science 291: 1284-1289.

Yu KD, Chen AX, Qiu LX, Fan L, et al. (2010). XRCC2 Arg188His polymorphism is not directly associated with breast cancer risk: evidence from 37,369 subjects. Breast Cancer Res. Treat. 123: 219-225.

Zhu H, Cao S, Liu Y, Ding X, et al. (2013). Genetic polymorphisms of xeroderma pigmentosum group D and prostate cancer risk: a meta-analysis. J. Cancer Res. Ther. 9: 187-192. 